\title{
Potencial de reutilización del efluente de la planta de tratamiento de agua residuales de Timaná- Huila para riego de Pasto Estrella (Cynodon plectostachyus)
}

\author{
Potential for the reuse of effluent from the Timaná-Huila \\ wastewater treatment plant for irrigation of star grass (Cynodon \\ plectostachyus)
}

\author{
Ana Milena Almario Ochoa ${ }^{1}$, Yury Samara Ortega Lincer ${ }^{2}$, Edinson Mujica \\ Rodriguez $^{3}$
}

\section{Resumen}

El tratamiento de las aguas residuales es una necesidad que tiene la sociedad para proteger su medio ambiente y garantizar el bienestar humano, por su parte el reúso de las aguas residuales tratadas es importante en la gestión integrada del recurso hídrico. En este estudio se realizó una revisión bibliográfica, recolección de información edafológica y climatológica, requerimientos hídricos y nutricionales del pasto estrella, y cálculo de la eficiencia de la Planta de Tratamiento de Aguas Residuales de Timaná. Según los resultados obtenidos en la investigación, se determinó que el efluente pertenece a la clase $\mathrm{C}_{2} \mathrm{~S}_{1}$, indicando que el agua es apta para riego, con peligro de salinidad medio y de alcalinidad baja. También, se estableció según las directrices de la Organización Mundial de la Salud que pertenece a la categoría B por su riesgo microbiológico y puede ser utilizada para riego del cultivo de pasto, lo que exige un adecuado monitoreo del riego para minimizar riesgos de salud pública. El área de estudio tiene déficit hídrico anual durante los meses de enero y febrero, y junio a septiembre. El efluente como riego para el cultivo de Pasto Estrella, puede irrigar en promedio 97 Has y aportar macronutrientes en las siguientes cantidades: Nitrógeno 19.634

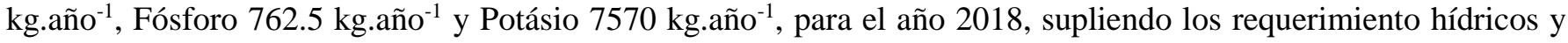
nutricionales al cultivo de pasto estrella.

Palabras Clave: requerimientos hídricos; requerimientos nutricionales; reúso; eficiencia; cultivo.

\begin{abstract}
The treatment of wastewater is a need that society has to protect the environment and ensure human well-being, while the treatment of treated wastewater is important in the integrated management of water resources. In this study, a bibliographic review, collection of soil and climatological information, hydrological and nutritional requirements of the star grass, and calculation of the efficiency of the Timaná Wastewater Treatment Plant were carried out. According to the results of the investigation, it was determined that the effluent belongs to the class $\mathrm{C} 2 \mathrm{~S} 1$, which indicates the water is suitable for irrigation, with danger of medium salinity and low alkalinity. The guidelines of the World Health Organization that belongs to category B are also indicated for their microbiological risk and that is the irrigation of the grass crop, which requires adequate monitoring of public irrigation. The study area has an annual water deficit during the months of January and February, and June to September. The effluent as irrigation for the cultivation of Star Grass, can irrigate on average 97 Has and contributed macronutrients in the following quantities: Nitrogen 19.634 kg.year-1, Phosphorus 762.5 kg.year-1 and potassium $7570 \mathrm{~kg}$.year-1, for the year 2018, supplying the hydric and nutritional requirements in the cultivation of star grass.

Keywords: water requirements; nutritional requirements; reuse; efficiency, cultivation.

\footnotetext{
${ }^{1}$ Ingeniera Agrícola, Universidad Surcolombiana Sede Pitalito.Email: almadelrio6_@ hotmail.com.

${ }^{2}$ Ingeniera Agrícola, Universidad Surcolombiana Sede Pitalito.Email: samaraortega0203_@hotmail.com.

${ }^{3}$ Maestro en Ingeniería Agrícola y uso Integral de Agua, Docente de Tiempo completo Universidad Surcolombiana Sede Pitalito, km 1 vía el Macal-

Pitalito. Email: edinson.mujica@usco.edu.co
} 


\section{INTRODUCCIÓN}

El tratamiento de las aguas residuales es una necesidad que tiene la sociedad para proteger su medio ambiente y garantizar el bienestar humano, pues estas aguas configuran un peligro potencial para la salud pública, ya que a través de ellas se pueden transmitir innumerables enfermedades, lo cual genera grandes impactos a la población y la economía de los países (Drechsel \& Evans, 2010).Una de las practicas más comunes de disposición final de las aguas residuales domesticas ha sido la disposición directa sin tratamiento a los cuerpos de agua superficiales y en el suelo (Plevich et al., 2012); sin embargo, la calidad de estas aguas puede generar dos tipos de problemas: la primera de salud pública, particularmente importantes en países tropicales por la alta incidencia de enfermedades infecciosas, cuyos agentes patógenos se dispersan en el ambiente de manera eficiente a través de las excretas o las aguas residuales crudas (Steffen et al., 2015), y el segundo son los problemas ambientales, por afectar la conservación o protección de los ecosistemas acuáticos y del suelo (Yi et al., 2011), lo que contribuye a la pérdida de valor económico del recurso y del medio ambiente y genera a su vez una disminución del bienestar para la comunidad ubicada aguas abajo de las descargas. El concepto de prevención y control de la contaminación de los cuerpos hídricos, involucra estrategias de manejo adecuado de aguas residuales que deben implementarse, en el contexto de la Gestión Integrada del Recurso Hídrico (Almeida et al., 2013). El reúso de agua residual en la agricultura es una de estas estrategias de prevención y control de la contaminación hídrica y es una alternativa para aumentar los recursos donde se presenta escasez de agua (Hanjra et al., 2012). De acuerdo con diferentes organizaciones internacionales aproximadamente el 90\% del agua residual en los países en vías de desarrollo se descarga sin tratamiento alguno a los cuerpos hídricos (UNEP, 2010). En Latinoamérica, sólo el 8\% del agua residual que se produce diariamente es tratada (Banco mundial et al., 2007) y es entregada a fuentes superficiales y cerca de 500.000 hectáreas son irrigadas, la mayoría con agua cruda (Madera, 2005).

Colombia tiene una superficie irrigada con aguas residuales de 1.230 .193 ha, con $27 \%$ de agua residual tratada y 73\% sin tratar, por lo general diluida con aguas superficiales (Minagricultura 2011). Al igual que sucede en toda América Latina, no se cuenta con información completa y confiable sobre el tema del reuso (Cepis, 2003) y solamente $8 \%$ del total de aguas residuales que se producen diariamente son tratadas (Banco mundial et al., 2007). A pesar de la alta capacidad de autopurificación, los ríos colombianos se encuentran en muchos casos contaminados en largos trayectos debido a la alta carga orgánica, de patógenos y de nutrientes que reciben. El riego agrega un tratamiento extra significativo a las aguas residuales, debido a la destrucción de compuestos refractarios en el suelo, evaporación de compuestos volátiles, muerte de patógenos, degradación de la materia orgánica remanente y otros procesos (Lopera \& Campos, 2011). La irrigación agrícola suele ser la alternativa más barata para la disposición final de las aguas servidas tratadas (comparada con la descarga a ríos, lagos u otras alternativas) tanto en costos de construcción como de operación y mantenimiento (Melián \& Fernández, 2016). Pérez y Hernández (2007) expresan algunas posibilidades que ofrece la utilización de aguas residuales en el riego de los cultivos las cuales contribuyen al aumento del $20 \%$ de la disponibilidad productos agrícolas frescos, ahorro del 10\% de fertilizante, debido al valor nutritivo de esta agua, y disminuye al $60 \%$ los gastos por concepto de transporte y otros insumos. El municipio de Timaná en el departamento del Huila cuenta con una planta de tratamiento de aguas residuales que vierte su efluente en el rio Timanejo creando problemas de contaminación ambiental y afectando comunidades aguas abajo que utilizan las aguas de este rio para consumo doméstico. Este estudio presenta el potencial de reutilización del efluente de la PTAR de Timaná como suministro de agua y nutrientes para el cultivo de pasto estrella, como una alternativa ambiental y productiva, considerada como un tratamiento terciario para la PTAR, donde el efluente pasa de ser un contaminante a convertirse en un recurso. El manejo integral del recurso hídrico implica la gestión eficaz basada en el ahorro, el reuso y la no contaminación del agua, todo ello en una planificación sostenible del territorio y una gestión integrada de las cuencas hidrográficas (Morató et al., 2006). 


\section{MATERIALES Y MÉTODOS}

La metodología está basada en el artículo propuesto por los ingenieros Eduardo valencia, Renso Alfredo Aragón y Jonathan Romero de la Universidad Surcolombiana, enfocados en el potencial de reutilización del efluente da la planta de tratamiento de aguas residuales de Nátaga en un cultivo de cacao (Theobroma cacao l.) realizado en el año 2012.

\section{Localización}

El municipio de Timaná se encuentra al sur del departamento del Huila a $166 \mathrm{~km}$ de la ciudad de Neiva, en el valle montañoso del Magdalena, subregión que corresponde a las estribaciones de las cordilleras central y occidental, tiene una extensión de 182.5 Kilómetros cuadrados, a una altura de 1100 y 2000 metros sobre el nivel del mar, con una temperatura media de $24^{\circ} \mathrm{C}$. El municipio de Timaná cuenta con una PTAR (Planta de tratamiento de aguas residuales), la cual se encuentra ubicada en las coordenadas $\mathrm{N}$ $01^{\circ} 59^{\prime} 12,64$ ", E $75^{\circ} 55^{\prime} 49,5^{\prime \prime}$, cuya cobertura es del $100 \%$ de la población del casco urbano y recibe un vertimiento de 24 L.s ${ }^{-1}$; la obra que fue ejecutada por la Sociedad de Acueducto, Aseo y Alcantarillado de Timaná, tuvo aportes del Gobierno Departamental y de la Corporación del Alto Magdalena, CAM y la construcción fue realizada por Aguas del Huila en el año 2009.

\section{Descripción general}

Se realizó la consulta del estado del arte, la cual consistió en revisión de artículos científicos y bases de datos sobre aguas residuales, reutilización de aguas residuales con fines de riego, requerimiento hídrico y nutricional del cultivo de Pasto Estrella (Cynodon plectostachius). Se recolectó información climatológica de la zona de estudio proporcionados por La Estación El Grifo ubicada en el municipio de Altamira Huila, como precipitación, evaporación, temperatura, humedad relativa, velocidad del viento. También se tomaron muestras de suelo en la zona donde está ubicado el cultivo de Pasto Estrella, para el análisis fisicoquímico de éste. De igual manera se hizo el reconocimiento y análisis del área de estudio con la recolección de información del diseño y monitoreo de la PTAR. Se tomaron datos e información sobre las unidades del sistema de tratamiento, población, caudales y disposición del efluente, de igual manera se tomaron y analizaron los resultados fisicoquímicos y microbiológicos para la caracterización del afluente; se realizaron muestreos puntuales del afluente y el efluente de la PTAR, el 28 de septiembre de 2017, en horas de la mañana y posteriormente se analizaron en los Laboratorios de Aguas Diagnosticamos del municipio de Neiva Huila y Construcsuelos suministros LTDA. Algunos de estos fueron: demanda biológica de oxígeno (DBO), demanda química de oxigeno (DQO), sólidos suspendidos (SS), grasas y aceites (G y A), nitrógeno $(\mathrm{N})$, Fósforo (P), Potasio (K), conductividad eléctrica (CE); microbiológicos se analizaron, coliformes totales (CT) y coliformes fecales (CF), algunos datos faltantes se tomaron de la literatura. Se determinó la eficiencia de la PTAR, lo que permitió la caracterización del efluente y su categorización como agua para riego, de acuerdo con las directrices de la FAO (1999) y de la OMS (1989). Para el cálculo de la eficiencia se empleó la ecuación número 1:

$$
\% R e=\frac{\text { Concentración } A f-\text { concentración } E f}{\text { concentración } A f} * 100
$$

La determinación del volumen de agua y nutrientes disponibles para reutilizar en la fertirrigación del cultivo de pasto estrella, se realizó con base en la población del municipio de Timaná, empleando el método geométrico para el cálculo de la población, con tasa de crecimiento de $0,56 \%$ y un periodo de 20 años. Se determinó el área a regar, por medio de la metodología de Jaramillo (2008) y los aportes en nutrientes: 
Nitrógeno $(\mathrm{N})$, fósforo $(\mathrm{P})$ y potasio $(\mathrm{K})$ para el cultivo de pasto estrella, mediante el cálculo de los recursos aportados por el efluente, en el año 2018; para esto se utilizaron las ecuaciones 2 y 3 :

$$
\begin{gathered}
\text { Cantidad de nutrientes }=\text { Población } * C a_{c p} \text { (2) } \\
\qquad a_{c p}=\operatorname{Dot} * C
\end{gathered}
$$

\section{RESULTADOS Y DISCUSIÓN}

\section{Descripción de la Planta de Tratamiento}

El municipio de Timaná en el 2016, contaba con 20350 habitantes y la población está distribuida de la siguiente manera: en la cabecera 7378 habitantes, que corresponde a 36\% y el restante 12.972 personas en la zona rural, correspondiente al 64\% (Plan de desarrollo de Timaná, 2016) y es de vocación agrícola. Cuenta con los servicios públicos básicos, la dotación estimada para el 2010 es 240 L.hab $^{-1}-\mathrm{d}^{-1}$ y se contempla una disminución gradual hasta $174 \mathrm{Lhab}^{-1}-\mathrm{d}^{-1}$, para el 2036.

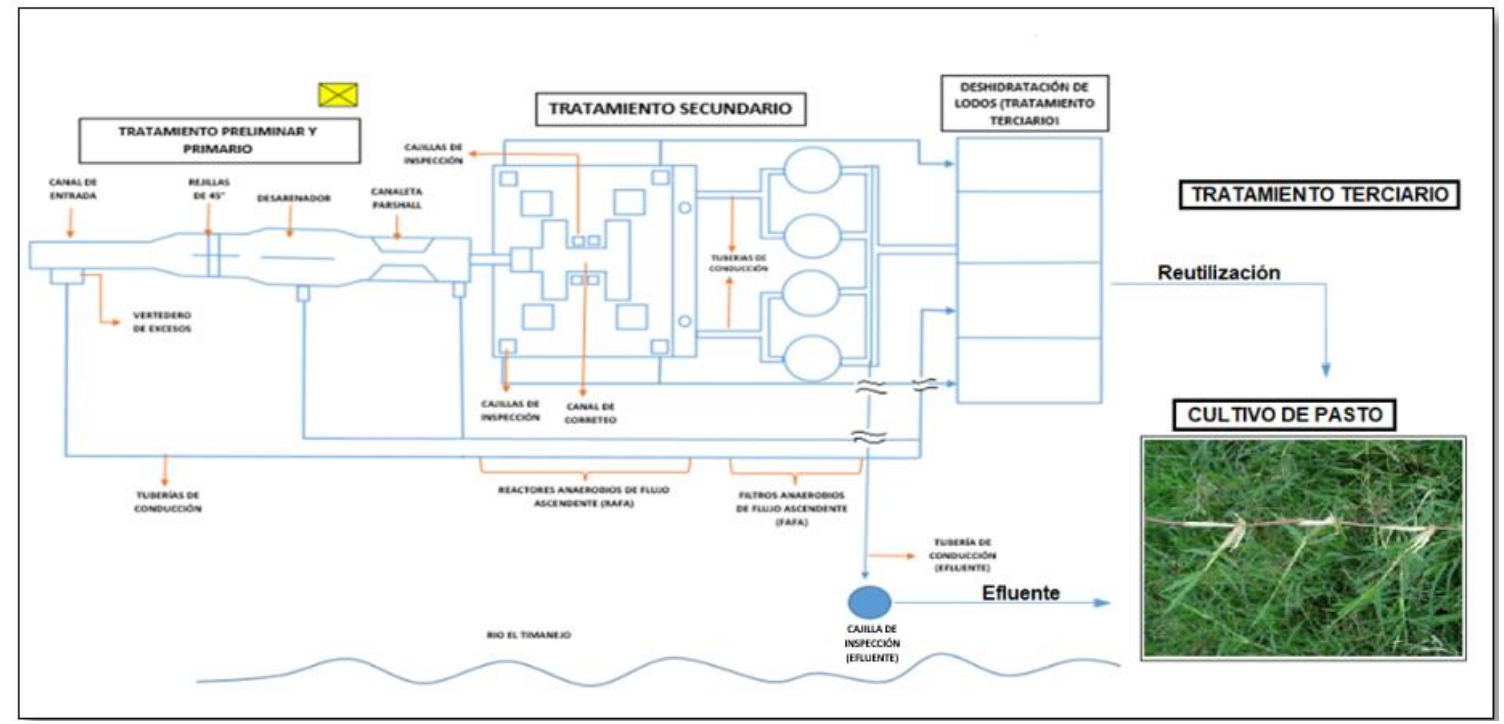

Figura 1. Esquema general de la PTAR del municipio de Timaná.

La PTAR tiene una cobertura del 100\% y recibe un caudal de 16.49 L.s ${ }^{-1}$. La figura 1 muestra el esquema general, donde se incluye la propuesta de reutilización del efluente en un cultivo de Pasto Estrella, la cual, está conformada por las siguientes unidades: Tratamiento preliminar y primario, consta de un aliviadero, un vertedero de excesos, dos rejillas con sus respectivos escurridores para residuos pesados y dos desarenadores en paralelo, una canaleta Parshall, por último, se encuentra ubicado un aliviadero y un trampa grasas; tratamiento secundario, conformado por cuatro UASB (Upflow anaerobic sludge blanket) y cuatro Filtros Anaerobios de Flujo Ascendente (FAFA), con lechos filtrantes plásticos y con cortes de guadua; los lodos son llevados a cuatro lechos de secado; actualmente, el efluente es vertido al rio Timanejo (Ver figura $1)$. 


\section{Análisis de suelos}

Propiedades químicas

Tabla 1. Análisis del contenido de nutrientes en el suelo

\begin{tabular}{|c|c|c|c|}
\hline NUTRIENTE & $\begin{array}{c}\text { CONTENIDO EN EL } \\
\text { SUELO }(\mathbf{k g . ~ h a}\end{array}$ & $\begin{array}{c}\text { REQUERIMIENTO DEL } \\
\text { CULTIVO (kg. ha }\end{array}$ & $\begin{array}{c}\text { DÉFICIT } \\
\left.\text { (kg. ha }^{\mathbf{- 1}}\right)\end{array}$ \\
\hline Nitrógeno & 67.32 & 230 & 162.68 \\
\hline Fósforo & 103.3 & 53 & - \\
\hline Potasio & 12.34 & 252 & $239 ., 6$ \\
\hline
\end{tabular}

Según los datos de la tabla anterior tomados del análisis de suelos y del requerimiento nutricional del cultivo, se logró establecer que la zona de estudio cuenta con un déficit de Nitrógeno y potasio de $162.68 \mathrm{~kg} \cdot \mathrm{ha} \mathrm{a}^{-1}$ y $239.6 \mathrm{~kg} \cdot \mathrm{ha^{-1 }}$ respectivamente, deficiencias que pueden ser suplidas con la cantidad de nutrientes aportados por el efluente de la PTAR el cual produce una cantidad de nitrógeno de $490 \mathrm{~kg} \cdot \mathrm{ha}^{-1}$ y $190.29 \mathrm{~kg} \cdot \mathrm{ha^{-1 }}$ de potasio. En cuanto al fósforo se puede observar que el contenido del suelo suple las necesidades del cultivo y se sugiere complementar la cantidad requerida de potasio puesto que esta no se suple con ninguno de los dos contenidos del nutriente (suelo y efluente).

\section{Propiedades físicas}

Para la zona de estudio, según los análisis de laboratorio se tiene una densidad aparente de 1,71 g.cc ${ }^{-1}$, lo que indica que es un suelo compactado y ésto puede presentarse debido a que son suelos utilizados para el pastoreo y el constante pisoteo de los rumiantes produce la compactación. En cuanto a la capacidad de intercambio de cationes (CC) y punto de marchites permanente (PMP) de la zona de estudio corresponde a $36.09 \%$ y $18.04 \%$ respectivamente.

\section{Evaluación del sistema}

Los resultados de la caracterización del Efluente y Afluente y la Eficiencia de la PTAR se presentan en la tabla 1. Estos datos fueron calculados de los datos obtenidos de los análisis de laboratorio de Diagnosticamos S.A. y Construcsuelos suministros LTDA.

\section{Calidad del efluente como agua para riego Calidad fisicoquímica}

Para determinar la calidad fisicoquímica del efluente de la PTAR de Timaná- Huila se tuvo en cuenta los siguientes parámetros: CE y RAS (El dato del Ras no fue calculado debido a la falta de datos, por lo que se tomó de la investigación Valencia del año 1998), cuyos valores corresponden a $0.30185 \mathrm{dS} / \mathrm{m}$ y 1.5 respectivamente. Según los parámetros antes mencionados y el Diagrama de clasificación de aguas para riego U.S. Salinity Laboratory Staff (1954), se determinó que el efluente es C2S1, lo que quiere decir que el agua es apta para riego, de buena calidad, con un peligro de salinidad medio y de alcalinidad bajo. 


\section{Calidad microbiológica}

Para determinar la calidad microbiológica del efluente como agua para riego, se tuvo en cuenta el valor de los $\mathrm{CF}=0,24 \times 10^{6} \mathrm{NMP} .100^{-1} \mathrm{~mL}^{-1}$ (fuente: Loboratorio Diagnosticamos) y las directrices de la OMS, con base en estos aspectos se determinó que el efluente pertenece a la categoría $\mathrm{B}$, siendo apto para el riego de cultivos de cereales, industriales, forrajeros, praderas y árboles.

Tabla 2. Caracterización del afluente y efluente- Eficiencias reales de la PTAR.

\begin{tabular}{|c|c|c|c|c|}
\hline \multicolumn{5}{|c|}{ RESULTADOS AÑO 2017} \\
\hline PARÁMETROS & UNIDADES & AFLUENTE & EFLUENTE & $\begin{array}{c}\% \\
\text { REMOCIÓN }\end{array}$ \\
\hline DBO5 & $\mathrm{Mg} / \mathrm{L}$ & 150.68 & 22 & 85.40 \\
\hline DQO & $\mathrm{Mg} / \mathrm{L}^{-1}$ & 240 & 10 & 95.83 \\
\hline GRASAS Y ACEITES & $\mathrm{Mg} / \mathrm{L}^{-1}$ & $<10$ & $<13$ & -130 \\
\hline FÓSFORO & $\mathrm{mg} \mathrm{P} / \mathrm{L}^{-1}$ & 4.7 & 1.5 & 68.09 \\
\hline POTASIO & $\mathrm{Mg} / \mathrm{L}^{-1}$ & 13.7 & 14.7 & -7.30 \\
\hline NITRÓGENO & $\mathrm{mg} \mathrm{N} / \mathrm{L}^{-1}$ & 15.5 & 37.9 & -144.52 \\
\hline CONDUCTIVIDAD & $\mu \mathrm{s} / \mathrm{cm}^{-1}$ & 242.7 & 301.85 & -24.37 \\
\hline $\begin{array}{c}\text { SÓLIDOS } \\
\text { SUSPENDIDOS } \\
\text { TOTALES }\end{array}$ & $\mathrm{Mg} / \mathrm{L}^{-1}$ & 284.0 & 13 & 95.42 \\
\hline $\begin{array}{c}\text { COLIFORMES } \\
\text { FECALES }\end{array}$ & $\begin{array}{l}\mathrm{NMP} / 100^{-} \\
{ }^{1} \mathrm{ml}^{-1}\end{array}$ & $0.024 * 10^{9}$ & $0.24 * 10^{6}$ & 99.00 \\
\hline $\begin{array}{l}\text { COLIFORMES } \\
\text { TOTALES }\end{array}$ & $\begin{array}{l}\mathrm{NMP} / 100 \\
\mathrm{ml}\end{array}$ & $0.024 * 10^{9}$ & $0.24 * 10^{6}$ & 99.00 \\
\hline
\end{tabular}

Fuente: Laboratorio Diagnosticamos y Construcsuelos suministros LTDA.

Las remociones de DBO del 85.40\%, DQO del 95.83\%, SS del 95.42\%, CF 99\%, y CT 99\% (Tabla 2), indican que la PTAR es eficiente en cuanto a la remoción de contaminantes, eficiencias que se pueden aumentar mejorando su operación; no obstante, en los parámetros de grasas y aceites, potasio, nitrógeno y conductividad, los valores son negativos ya que la toma de muestras de las aguas residuales se hizo en diferentes fechas y es por esto que los valores emitidos por el laboratorio Diagnosticamos presentan un margen de error.

\section{Caudal del efluente de la PTAR}

Se tomó como valor de caudal, el dato correspondiente al promedio de los aforos realizados en la PTAR, el día 31 de 03 del 2017. Los aforos se realizan diariamente en la canaleta Parshall.

$$
Q_{A R}=16.42 \mathrm{Lps}
$$

Para obtener el caudal futuro, se determinó la población futura y se utilizó el modelo geométrico (RAS, 2000) basado en los datos obtenidos por el DANE en los censos realizados. Definido por la ecuación 4.

$$
P=P_{A} *(1+r)^{n}(4)
$$


Se proyectó la población al año 2018 reemplazando en la ecuación 4 los datos correspondientes a la población del año 2017 que es de 7378 habitantes (PD TIMANÁ, 2016), la tasa de crecimiento de 0,56\% según el PD TIMANÁ para el año 2016 y el periodo proyectado a 2 años (2017 a 2018), de la siguiente manera:

$$
P=7378 *(1+0.0056)^{2}=7461 \text { hab }
$$

También se calculó la muestra de la dotación de agua potable del municipio teniendo en cuenta el QAR de los aforos de la PTAR y la población del año 2018, mediante la ecuación 5:

$$
\begin{gathered}
\text { Dotación }=\frac{Q_{A R}}{\text { Población } * C R}(5) \\
\text { Dotación }=\frac{1418688 \text { L.día }{ }^{-1}}{7461 \text { hab } * 0.8}=237 \text { L. }(\text { hab }- \text { dia })^{-1}
\end{gathered}
$$

El caudal de agua residual (Q $\left.\mathrm{QR}_{\mathrm{AR}}\right)$ para el año 2018 se calculó mediante la ecuación 6:

$$
\begin{gathered}
Q_{A R}=P_{A} * \operatorname{Dot}^{*} 0.8(6) \\
Q_{A R}=7.461 \mathrm{hab} * 0027430 \mathrm{Lps} * 0.8=16,3 \mathrm{Lps}
\end{gathered}
$$

\section{Nutrientes aportados por el efluente}

\section{- Cargas Per Cápita en el Efluente}

Para el cálculo del aporte de los nutrientes: Nitrógeno (N), Fosforo (P) y Potasio (K), de la PTAR se tuvieron en cuenta los resultados de los análisis de agua del efluente emitidos por el Laboratorio Diagnosticamos. Se multiplicó la dotación de agua potable por el coeficiente de retorno (0.8) y por la cantidad del nutriente aportado por el efluente, así:

$$
C a_{p c}=\text { Dotación } * 0.8 * \text { Nut.ef }(7)
$$

Muestra de cálculo para Nitrógeno en el año 2018:

$$
C a_{p c} N=237237 \text { L. }(\text { hab }- \text { dia })^{-1} * 0,0379 \text { gr. } L^{-1} * 0,8=6,54 \text { gr. }(\text { hab }- \text { dia })^{-1}
$$

Tabla 3. Cargas Per cápita de Nutrientes en el Efluente para el año 2018

\begin{tabular}{|c|c|c|}
\hline Parámetro & $\begin{array}{c}\text { Nutrientes } \\
\text { aportados por el } \\
\text { efluente }\left(\mathbf{m g} / \mathbf{L}^{-\mathbf{1}}\right)\end{array}$ & Capc $\left(\mathbf{g} / \mathbf{h a b}^{\mathbf{- 1}} \mathbf{- d}^{\mathbf{- 1}}\right)$ \\
\hline $\mathbf{N}$ & 37.9 & 7.18 \\
\hline $\mathbf{P}$ & 1.5 & 0.28 \\
\hline K & 14.7 & 2.78 \\
\hline
\end{tabular}

Los contaminantes para el ambiente $\mathrm{N}, \mathrm{P}$ y K, en la reutilización se convierten en un recurso que puede suplir en parte las necesidades de nutrientes de las plantas (Lopera \& Campos, 2011). En la tabla 2 se muestra el aporte de nutrientes por parte del efluente de la PTAR al cultivo. 


\section{Cantidad de nutrientes}

Se procedió a calcular la cantidad de nutrientes aportados por el efluente en el año 2018. A continuación, se presenta la muestra de cálculo para el nitrógeno, calculada con la ecuación 2:

Cantidad $N=7.461 \mathrm{hab} * 2.62 \mathrm{~kg} \cdot(\text { hab.año })^{-1}=19553 \mathrm{Kg} * a \tilde{\mathrm{n}} o^{-1}$

Tabla 4. Cantidad de Nutrientes aportados por el Efluente.

\begin{tabular}{|c|c|}
\hline Nutriente & ${\text { Cantidad }\left(\text { Kg.año }^{-1}\right)}^{-1}$ N \\
\hline $\mathbf{P}$ & 79553 \\
\hline K & 763 \\
\hline
\end{tabular}

\section{Requerimiento nutricional para el cultivo de Pasto Estrella}

Tabla 5. Requerimientos nutricionales del Pasto Estrella (Cynodon plectostachius).

\begin{tabular}{|c|c|c|c|c|}
\hline \multirow{2}{*}{ Variedad de pasto } & \multirow{2}{*}{$\begin{array}{l}\text { Producción de } \\
\text { materia seca } \\
\left.\text { (ton.ha' }{ }^{-1} \text { año }{ }^{-1}\right)\end{array}$} & \multicolumn{3}{|c|}{$\begin{array}{l}\text { Extracción de nutrientes } \\
\left(\mathrm{Kg.ha}^{-1} \mathbf{a n ̃ o}^{-1}\right)\end{array}$} \\
\hline & & $\mathbf{N}$ & $\mathbf{P}$ & $\mathbf{K}$ \\
\hline Cynodon Plectostachius & 19 & 230 & 53 & 252 \\
\hline
\end{tabular}

Fuente: Bernal \& Espinoza (2003).

\section{Requerimientos Hídricos}

El requerimiento hídrico del cultivo de Pasto Estrella se determinó con el cálculo de la demanda de riego neta mensual multianual de los años 2005 al 2015, haciendo uso de los valores de precipitación efectiva, evapotranspiración y uso consuntivo (Ver tabla 6).

Tabla 6. Demanda de Riego mensual multianual (2005-2015)

\begin{tabular}{|l|c|c|c|c|}
\hline \multicolumn{1}{|c|}{ MESES } & $\begin{array}{c}\text { PRECIPITACIÓN } \\
\text { EFECTIVA (mm/mes }\end{array}$ & $\begin{array}{c}\text { EVAPORACIÓN } \\
\left(\mathbf{m m} / \mathbf{m e s}^{-\mathbf{1}}\right)\end{array}$ & $\begin{array}{c}\text { USO CONSUNTIVO } \\
\left(\mathbf{m m} / \mathbf{m e s}^{-\mathbf{1}}\right)\end{array}$ & $\begin{array}{c}\text { DEMANDA DE } \\
\text { RIEGO NETA } \\
\left(\mathbf{m m} / \mathbf{m e s}^{-\mathbf{1}}\right)\end{array}$ \\
\hline Enero & 67.23 & 97.76 & 92.90 & -25.60 \\
\hline Febrero & 90.04 & 74.56 & 70.80 & 19.20 \\
\hline Marzo & 108.18 & 123.44 & 117.30 & -9.10 \\
\hline Abril & 105.94 & 82.32 & 78.20 & 27.70 \\
\hline Mayo & 111.90 & 81.20 & 77.10 & 34.80 \\
\hline Junio & 88.47 & 97.92 & 93.00 & -4.60 \\
\hline Julio & 74.39 & 84.48 & 80.30 & -5.90 \\
\hline Agosto & 61.90 & 92.00 & 87.40 & -25.50 \\
\hline Septiembre & 46.09 & 94.24 & 89.50 & -43.40 \\
\hline Octubre & 105.91 & 92.24 & 87.60 & 18.30 \\
\hline Noviembre & 104.62 & 97.52 & 92.60 & 12.00 \\
\hline Diciembre & 110.84 & 73.44 & 69.80 & 41.10 \\
\hline
\end{tabular}


La tabla 6 muestra valores negativos en la demanda neta de riego, lo indica que en estos meses se requiere riego equivalente a cada uno de los valores. Demanda de riego neta: es la cantidad de agua que se debe aplicar en un riego (Jaramillo, 2008); se calcula para cada mes, mediante la ecuación 8:

$$
D R n=P e-U . C(8)
$$

Muestra de cálculo para el mes de Enero:

$D R n=67,23 \frac{\mathrm{mm}}{\mathrm{mes}}-92,9 \mathrm{~mm} / \mathrm{mes}$

$D R n=-25,6 \mathrm{~mm} / \mathrm{mes}$

En la Figura 2 se puede observar que hay un déficit hídrico leve en el primer semestre del año y se presenta en los meses de enero y marzo. También se puede determinar el déficit en el segundo semestre del año en los meses de junio hasta octubre, siendo el mes de septiembre el más crítico con un déficit $-43,4 \mathrm{~mm}$. Los valores de evapotranspiración y precipitación efectiva se presentan en la Tabla 5.

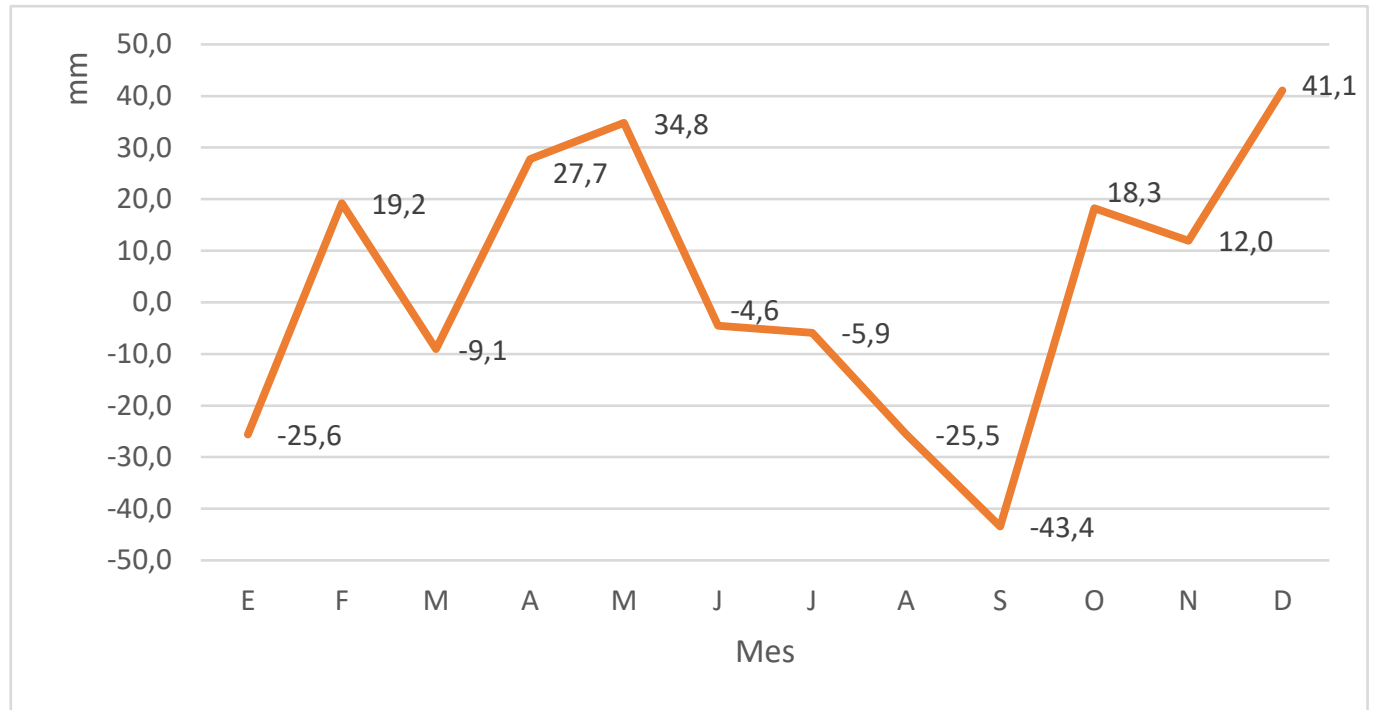

Figura 2. Demanda de Riego Neta (mm/mes) para el cultivo de Pasto Estrella.

\section{- Necesidad de Riego}

De acuerdo con el análisis del balance hídrico, se determinó la necesidad de riego teniendo en cuenta el dato representativo para el mes más crítico (verano) que en este caso es septiembre, el cual presenta una demanda de riego neta de $-43,4 \mathrm{~mm}$ (Ver Tabla 6).

Esta demanda en mm se puede expresar en unidades de caudal, para un flujo o gasto continuo, mediante la ecuación 9 (Jaramillo, 2008):

$$
Q R n=\frac{0.116 * D R n * A}{30}
$$

Se despeja el área de la ecuación 9, para determinar el número de hectáreas que se puede irrigar en época de estiaje: 


$$
\begin{gathered}
A=\frac{30 * Q R n}{0.116 * D R n}(10) \\
A=\frac{30 * 16.3}{0.116 * 43,4}=97 \text { has }
\end{gathered}
$$

Con un caudal de 16.3 Lps, teniendo en cuenta el mes más crítico del año, que para este caso es el mes de septiembre con un déficit de $43.4 \mathrm{~mm}$, se logra regar un área de 97 has.

\section{Potencial de reutilización de nutrientes}

En el potencial de reutilización de los nutrientes se hizo una comparación entre los nutrientes aportados por el efluente y los nutrientes requeridos por el cultivo, para el año 2018, en época de verano, con la ecuación 11:

$$
\operatorname{ReN}\left(k g \cdot a \tilde{\mathrm{n}} o^{-1}\right)=\operatorname{ReN}\left(\mathrm{kg} \cdot\left(h a-a \tilde{\mathrm{n}} o^{-1}\right)\right) * N o \cdot \text { Has }
$$

Muestras de cálculo para el Requerimiento de Nitrógeno (N), para el año 2018 en época de verano:

$$
\operatorname{ReN}\left(k g \cdot a \tilde{\mathrm{n}} o^{-1}\right)=230 * 97=22310 \mathrm{~kg} \cdot a \tilde{\mathrm{n}} o^{-1}
$$

El porcentaje de nutrientes suplidos por el efluente se calculó con la ecuación 12, donde el aporte del efluente en $\mathrm{kg}$. $a \tilde{n} o^{-1}$ se tomó de la Tabla 4 y el requerimiento del cultivo se tomó de la Tabla 5.

$$
\% N_{\text {suplido }}=\frac{\text { Aporte Efluente }\left(\mathrm{kg} \cdot a \mathrm{n} o^{-1}\right)}{\text { Requerimiento cultivo }\left(\mathrm{kg} \cdot a \mathrm{n} o^{-1}\right)} * 100
$$

Muestra de cálculo para Nitrógeno en el año 2018 época de verano:

$$
\% N_{\text {suplido }}=\frac{19553\left(\mathrm{~kg} \cdot a \tilde{\mathrm{n}} o^{-1}\right)}{22310\left(\mathrm{~kg} \cdot a \tilde{\mathrm{n}} o^{-1}\right)} * 100=88 \%
$$

Tabla 6. Potencial de reutilización de nutrientes para el año 2018.

\begin{tabular}{|c|c|c|c|}
\hline Nutriente & $\begin{array}{c}\text { Requerimiento } \\
\left(\text { Kg.año }^{-1}\right)\end{array}$ & $\begin{array}{c}\text { Aporte del } \\
\text { efluente }\left(\mathbf{K g . a n ̃ o}^{-1}\right)\end{array}$ & $\begin{array}{c}\text { Porcentaje } \\
\text { Suplido (\%) }\end{array}$ \\
\hline $\mathbf{N}$ & 22310 & 19553 & 88 \\
\hline $\mathbf{P}$ & 5141 & 763 & 15 \\
\hline K & 24444 & 7570 & 31 \\
\hline
\end{tabular}

Según la tabla 6 se logró establecer que el aporte de nutrientes por parte del efluente es deficiente para la fertilización del cultivo de pasto estrella con respecto al fosforo $(\mathrm{P})$ y el potasio $(\mathrm{K})$.

\section{CONCLUSIONES}

El efluente de la PTAR del municipio de Timaná, por su calidad como agua para riego pertenece a la clase $\mathrm{C} 2 \mathrm{~S} 1$, indicando que el agua es apta para riego, de buena calidad, con un peligro de salinidad medio y de alcalinidad bajo. También se logró establecer según las directrices de la OMS que el efluente 
pertenece a la categoría B por su riesgo microbiológico y puede ser utilizada para riego del cultivo de pasto estrella (Cynodon Plectostachius), lo que exige un adecuado monitoreo del riego para minimizar posibles riesgos de salud pública.

Con el efluente de la PTAR del municipio de Timaná, en la temporada de verano se pueden regar en promedio 97 Has (para el año 2018) de pasto estrella, por lo que esta propuesta de reutilización se convierte en una alternativa de reducción de la contaminación al medio ambiente, productiva por la fertirrigación de cultivos y al mismo tiempo funciona como tratamiento terciario para las aguas residuales, reduciendo el índice de contaminación del rio Timanejo y mejorando la calidad de vida de la población que reside aguas abajo del vertimiento.

También se logró establecer que el suelo cuenta con un déficit de nitrógeno y potasio de $162.68 \mathrm{Kg}$.año ${ }^{-1}$ y $239.7 \mathrm{Kg}$. año $^{-1}$, respectivamente, por lo que se recomienda suplir estas deficiencias con los nutrientes que contiene el efluente de la PTAR. La zona de influencia de la PTAR tiene déficit hídrico durante los meses de enero, mayo y de junio a septiembre; el efluente se constituye en un recurso potencial para el cultivo de pasto, aportando macronutrientes N (19.634 kg. año $\left.{ }^{-1}\right), \mathrm{P}\left(762.5 \mathrm{~kg} \cdot \mathrm{año}^{-1}\right), \mathrm{K}\left(7570 \mathrm{~kg} \cdot \mathrm{año}{ }^{-1}\right)$.

\section{RECOMENDACIONES}

En cuanto a los suelos de la zona de estudio se recomienda mejorar el sustrato con la aplicación de abonos orgánicos, debido a que estos presentan un bajo contenido de materia orgánica. Se recomienda implementar riego por superficie o aspersión al cultivo de pasto, teniendo un cuidado especial con el manejo de material grueso que pueda contener el efluente para prevenir que se produzca obstrucción en las boquillas del sistema.

Se sugiere tener en cuenta para una adecuada reutilización del efluente de la PTAR de Timaná en riego de pasto Estrella, la realización de estudios necesarios donde se muestre la asimilación de nutrientes por parte de las plantas, así como también estudios de suelos para evaluar el comportamiento del suelo en cuanto a la fertirrigación con dicho efluente.

\section{Nomenclatura}

\%Re: Porcentaje de remoción (\%)

Af: Afluente

Ef: Efluente

$\mathrm{Ca}_{\text {cp: }}$ Carga per-cápita de $\left(\mathrm{g}_{\text {.hab }}{ }^{-1}-\right.$ día $\left.^{-1}\right)$

L: Litro

Hab: Habitantes

Dot: Dotación (L.hab ${ }^{-1}$-día ${ }^{-1}$ )

C: Concentración de nutriente (mg N.L ${ }^{-1}$ )

P: Población futura (habitantes)

$\mathrm{P}_{\mathrm{A}}$ : Población actual (habitantes)

R: Tasa de crecimiento

n: Periodo proyectado

QAR: Caudal de agua residual (L.s ${ }^{-1}$ )

$\mathrm{S}$ : segundo

CR: Coeficiente de retorno $(0,8)$

Nut.ef: Nutrientes aportados por el efluente (g) 
QRn: Caudal de riego neto (L.s $\left.{ }^{-1}\right)$

DRn: Demanda de riego neto $\left(\mathrm{L} . \mathrm{s}^{-1}\right)$

$\mathrm{A}_{\mathrm{I}}=$ Área a Irrigar $\left(\mathrm{m}^{2}\right)$

$\mathrm{ReN}=$ Requerimiento del nutriente $\left(\mathrm{Kg} \mathrm{Ha}^{-1}-\mathrm{año}^{-1}\right)$

No. Has $=$ Número de hectáreas $(\mathrm{Ha})$

$\% \mathrm{~N}_{\text {suplido: }}$ Porcentaje de nutriente suplido (\%)

\section{REFERENCIAS BIBLIOGRÁFICAS}

Alcaldía municipal de Timaná, 2016. Plan de desarrollo municipio de Timaná. http://timanahuila.micolombiadigital.gov.co/sites/timanahuila/content/files/000047/2306_plan-dedesarrollo-municipio-de-timana-periodo-20162019-porque-timana-somos-todos.pdf. Consultado: 27 noviembre de 2017. Consultado el 2 septiembre de 2017.

Almeida, G., Vieira, J., Marques, A., Kiperstok, A., \& Cardoso, A. 2013. Estimating the potential wáter reuse based on fuzzy reasoning. Journal of Environmental Management, 128, pp. 883-892. doi: 10.1016/j.jenvman.2013.06.048

Banco Mundial, Agencia Suiza para el Desarrollo y la Cooperacion (Cosud), Unicef y Banco Interamericano para el Desarrollo (Bid). 2007. Saneamiento para el desarrollo. Como estamos en 21 paises de America Latina y el Caribe. Conferencia Latinoamericana de Saneamiento, Cali.

Bernal, J., \& Espinoza, J. (2003). Manual de nutrición y fertilización de pastos. International Plant Nutrition Institute. Recuperado de https://www.yumpu.com/es/document/view/14237075/manualde-nutricion-y- fertilizacion-de-pastos-manual-. Consultado el 2 febrero de 2017. Cepis (Centro Panamericano de Ingenieria Sanitaria y Ciencias del Ambiente). 2003. Inventario de la situación actual de las aguas residuales domesticas en Colombia. Sistemas integrados de tratamiento y uso de aguas residuales en América Latina: realidad y potencial. Peru. En: www.cepis.ops.oms.org; consultado septiembre de 2017.

Drechsel, P. y Evans, A. E. V. 2010. Wastewater use in irrigated agriculture. Irrigated and Drainage Systems, Vol. 24, N. ${ }^{\circ}$ 1, pp. 1-3. Doi: 10.1007/s10795-010-9095-5

FAO (Food and Agriculture Organization of the United Nations). 1999. Wastewater treatment and use in agriculture. Recuperado de: www.fao.org/docrep/T0551E/T0551E00.htm.

Hanjra, M., Blackwell, J., Carr, G., Zhang, F. \& Jackson, T., 2012. Wastewater irrigation and environmental health: Implications for water governance and public policy. International Journal of Hygiene and Environmental Health, Vol. 215, No.3, pp. 255-269. Doi: 10.1016/j.ijheh.2011.10.003

Jaramillo, J.B., 2008. Curso: Riegos y Drenaje Notas de Clase. Palmira: Universidad Nacional de Colombia sede Palmira.

Madera, C., 2005. Reusó de Agua Residual: los Aspectos sobre Calidad Necesitan Mayor Atención. Consultado el 31 de Octubre de 2017. <http://www.es.irc.nl/page/34590>.

Melián, N., Fernandez, M., 2016. Reutilización de agua para la agricultura y el medioambiente, DOI 10.17561/at.v0i8.3298. 
Lopera, M., Campos, S., (2011). Proyecto: Desarrollo de capacidades en el uso seguro de aguas residuales para agricultura.

http://www.ais.unwater.org/ais/pluginfile.php/356/mod_page/content/128/Colombia_Informe\%20Naci onal.pdf. Consultado 1 enero de 2018.

Minagricultura (2011). Proyecto: Desarrollo de capacidades en el uso seguro de aguas residuales para agricultura.

http://www.ais.unwater.org/ais/pluginfile.php/356/mod_page/content/128/Colombia_Informe\%20Nacion al.pdf. Consutado 30 noviembre de 2016.

Melián, N., Fernandez, M., 2016. Reutilización de agua para la agricultura y el medioambiente, DOI 10.17561/at.v0i8.3298. https://revistaselectronicas.ujaen.es/index.php/atma/article/view/3298. Consultado el 18 junio de 2018.

Morató, J., Subirana A., Gris A., Carneiro A., Pastor R., 2006. Tecnologías Sostenibles para la Potabilización y el Tratamiento de aguas residuales. Revista Lasallista de investigación.

OMS (Organización Mundial de la Salud). (1989). Directrices sanitarias sobre el uso de aguas residuales en agricultura y acuicultura. Serie Informes técnicos, 778. Organización Mundial de la Salud (OMS), Ginebra. 93 p.

Pérez, J.; Hernández G. 2007. "Valoración de la calidad del agua del arroyo Guachinango con fines de riego", Revista Ciencias Técnicas Agropecuarias, 16(3): 6-9.

Plevich, J. O.; Delgado, A. R. S.; Saroff, C.; Tarico, J. C.; Crespi, R. J.; Barotto, O. M. 2012. El cultivo de alfalfa utilizando agua de perforación, agua residual urbana $\mathrm{y}$ precipitaciones, http://dx.doi.org/10.1590/S1415-43662012001200013.

RAS, 2000. Reglamento Técnico de Agua Potable y Saneamiento Básico. Disponible desde Internet en http://www1.minambiente.gov.co/viceministerios/ambiente/dir_agua_potable_saneam_basico/direccion/d ireccion_agua_potable.htm (con acceso el 18/10/2017).

Steffen, W.; Richardson, K.; Rockström, J.; Cornell, S. E.; Fetzer, I.; Bennett, E. M.; Biggs, R.; Carpenter, S. R.; De Vries, W.; De Wit, C. A.; Folke, C.; Gerten, D.; Heinke, J.; Mace, G. M.; Persson, L. M.; Ramanathan, V.; Reyers, B. y Sorlin, S. 2015. Planetary boundaries: Guiding human development on a changing planet. Science, Vol. 347, N. ${ }^{\circ}$ 6223. Doi: 10.1126/science. 1259855

UNEP. (2010). SICK WATER? The central rol of wastewater management in sustainable development. E. Corcoran, C. Nellemann, E. Baker, R. Bos, D. Osborn, H. Savelli, ed.

Yi, L., Jiao, W., Chen, X., \& Chen, W. (2011). An overview of reclaimed water reuse in China. Journal of Environmental Sciences, 23 (10), pp. 1585-1593. doi:10.1016/S1001-0742(10)60627-4 